\title{
Molecular cloning and analysis of a receptor-like promoter of Gbvdr3 gene in sea island cotton
}

\author{
B.-J. Zhang ${ }^{1,2 *}$, H.-P. Zhang ${ }^{2 *}$, Q.-Z. Chen ${ }^{1}$, N. Tang ${ }^{1}$, L.-K. Wang1, \\ R.-F. Wang ${ }^{2}$ and B.-L. Zhang ${ }^{3}$ \\ ${ }^{1}$ Nanjing Xiaozhuang University, Nanjing, China \\ ${ }^{2}$ College of Life Sciences, Anhui Agricultural University, Hefei, China \\ ${ }^{3}$ Institute of Agro-biotechnology, Jiangsu Academy of Agricultural Sciences, \\ Nanjing, China \\ *These authors contributed equally to this study. \\ Corresponding authors: Q.Z. Chen / R.F. Wang \\ E-mail: qzchen65@hotmail.com /rfwang@ahau.edu.cn
}

Genet. Mol. Res. 15 (2): gmr.15028636

Received March 23, 2016

Accepted April 11, 2016

Published May 23, 2016

DOI http://dx.doi.org/10.4238/gmr.15028636

\begin{abstract}
Verticillium wilt caused by soil borne fungus Verticillium dahliae could significantly reduce cotton yield. The Vel homologous gene Gbvdr3 is resistant to Verticillium wilt. In order to understand of the function of the promoter Gbvdr3 in Gossypium barbadense, the promoter region of the receptor-like gene Gbvdr3 was obtained by genome walking, and the cis-element in the promoter was identified using the PLACE software in this study. The sequence analysis showed that the promoter contained elements related to stress resistance and light regulation. The cloned promoter was fused to the GUS reporter gene and transformed into Arabidopsis. GUS expression was specifically detected in roots, flowers, and seeds, suggesting that the expression of $G b v d r 3$ is tissue-specific. Separation and characterization analysis of the promoter of $G b v d r 3$ provides a platform for further research and application of this gene. Thorough understanding of the function of the Gbvdr3 promoter is important for better understanding of Gbvdr3 function. These results indicated that the promoter of $G b v d r 3$ was a tissue-specific promoter.
\end{abstract}

Key words: Cotton; Receptor-like protein; Promoter; GUS staining 


\section{INTRODUCTION}

The leucine-rich repeat receptor-like protein kinase (LRR-RLK) belongs to the trans-membrane receptor-like kinases in plants. It is made up of leucine-rich repeats (LRRs) domain, a trans-membrane (TM) domain, and a functional protein kinase domain. As a signal recognition receptor, the LRR-RLK participates in signal transduction process of CLAVATA $(C L V)$, Brassinosteroid and Activator of Xa21 in different plants, and plays important roles in plant development and cell growth, hormone signaling, and stress responses (Anne and Steven, 2004). The biological function of these genes includes disease resistance response, stress resistance response, and plant morphogenesis. LRR-RLK such as rice OsSIK1 (Ouyang et al., 2010) and Arabidopsis ARCKI (Tanaka et al., 2012) and GHRI (Hua et al., 2012) play an important role in optimizing plant tolerance to abiotic stress.

The LRR-RLK participates in a diverse range of processes in response to abiotic stress (Huang et al., 2012) and disease resistance (Zhang et al., 2012). Many studies reported LRR-RLK genes that are involved in plant disease resistance response such as tomato Verticillium wilt resistance genes Vel and Ve2 (Kawchuk et al., 2001; Fradin et al., 2011), tomato leaf mold resistance gene $C f-9$ (Jones et al., 1994), tobacco and tomato Trichoderma viride resistance gene LeEIX2 (Ron and Avni, 2004), apple scab resistance genes HcrVfal and HcrVfa2 (Belfanti et al., 2004; Malnoy et al., 2008), and Arabidopsis genes AtRLP52 and AtRLP30 (Ramonell et al., 2005). In addition to the resistance in plants, these genes are also involved in organ development, morphogenesis, and so on. Thus, Arabidopsis CLV2 participates in the formation of shoot tips and root meristem, corn FEA2 maintains the apical meristem (Taguchi-Shiobara et al., 2001), and Arabidopsis TMM participates in control of stomatal distribution and development of stomata (Nadeau and Sack, 2002). Three Vel homologous genes, GbVe, GbVel and Gbvdr5, have been isolated from G. barbadense, and their resistance to various $V$. dahliae strains has been confirmed (Chen et al., 2016). In this study, a promoter of the receptor-like $G b v d r 3$ was cloned from a disease-resistant cultivar of Gossypium barbadense L. and transferred into Arabidopsis, creating transgenic plants with Gbvdr3 promoter.

\section{MATERIAL AND METHODS}

\section{Cloning the Gbvdr3 promoter}

Gbvdr3 promoter was isolated from the G. barbadense 'Hai7124' genomic DNA by PCR using primers P1 5'-CAGCTTATCAAACGCAGAAGGAA-3' and P2 5'-CACTTTGACATTGAGCCGAAACC-3'. Taq, buffer, and DNTPs were purchased from SenBeiJia Biological Technology Co., Ltd. (Nanjing, China). The PCR mixture included 15 $\mu \mathrm{L} 2 \mathrm{X}$ GC buffer I, $5 \mu \mathrm{L} 2.5 \mathrm{mM}$ DNTPs, $1.5 \mathrm{U}$ Taq enzyme, and approximately $1 \mathrm{mg} \mathrm{H} 7124$ template genomic DNA, and deionized water was added to make a final volume of $30 \mu \mathrm{L}$. The upstream sequence of $G b v d r 3$ gene was obtained by genome walking according to the Genome Walker ${ }^{\mathrm{TM}}$ (Clontech) instruction. The PCR products were purified from $1.0 \%$ agarose gels and then ligated into pGEMT vector (Promega, Madison, WI, USA) and transformed into DH5 $\alpha$ bacterial cells. The clones were confirmed by restriction enzyme digestion and sequencing. The Gbvdr3 promoter sequences were analyzed by the PLACE software (http:// www.dna.affrc.go.jp/htdocs/PLACE/). 


\section{Plasmid construction and Arabidopsis transformation}

To construct the vector, the entire coding region of $G b v d r 3$ was amplified by PCR using SamI and BamHI linker primers (Gbvdr3: 5'-TGAGTCGACCACAGAAGTTGAACAAA CTAGGCAT-3' and 5'-TGAGGATCCAGATAACAATGGCACTAGGTTGA-3') and ligated into the vector $\mathrm{pBI121}$. A 1200-bp fragment of the Gbvdr3 promoter was used to construct the pBI-Gbvdr3-GUS vector. This fragment was amplified by PCR and ligated into the vector. The transformation of female gametes of Arabidopsis is accomplished by simply dipping developing inflorescences for a few seconds into a 5\% sucrose solution containing 0.01-0.05\% Silwet L-77 (v/v) and resuspended agrobacterium cells carrying the genes to be transferred. Treated plants were allowed to set seeds, which were then plated on a selective medium to screen for transformants.

\section{Growth conditions and plant transformation}

Cotton seeds of Hai7124, a Verticillium wilt-resistant cultivar widely used in China for genetic and breeding studies, were delinted with concentrated sulfuric acid. Wild type Col-0 and transgenic plants were grown in potting soil or on half-strength Murashige-Skoog $(1 / 2 \mathrm{MS})$ medium under $120 \mu \mathrm{mol} \cdot \mathrm{m}^{-2} \cdot \mathrm{s}^{-1}$ light in a growth room at a temperature between $22^{\circ}$ and $24^{\circ} \mathrm{C}$, under a $16 \mathrm{~h}$ light $/ 8 \mathrm{~h}$ dark photoperiod, and $65 \%$ relative humidity. The identified transgenic plants and control plants were allowed to self-fertilize, and the resulting progenies were planted for further use in subsequent experiments.

\section{Histochemical and fluorometric GUS assays}

For histochemical staining of GUS, fresh tissue samples were obtained from Arabidopsis plants and immediately exposed to X-Gluc solution. A fluorometric GUS assay was performed as described by Jefferson et al. (1987). The fluorescence of 4-methylumbelliferone (4-MU), the product of GUS-catalyzed hydrolysis, was measured using the TECAN GENios system (Tecan Group Ltd., Mannedorf, Switzerland). Protein concentration in the supernatant was assessed by the method described by Bradford (1976), using bovine serum albumin as a standard. GUS activity was normalized to the protein concentration of each supernatant extract and calculated as molar unit $(\mathrm{M})$ of soluble protein per minute.

\section{Expressions of $G b v d r 3$ gene in different organs and stages of $G$. barbadense development}

Quantitative reverse transcription-PCR (qRT-PCR) for $G b v d r 3$ was conducted using a SYBR Premix ExTaqTM II Kit (TaKaRa, Shiga, Japan), the primer pair 5'-TCAGGA TTAAGTAGGGAAGGAGTT-3' and 5'-GTAATACAAGGTGGAAACAGAAGC-3', and cotton polyubiquitin gene UBQ14 (Artico et al., 2010) as the internal standard. The PCR program consisted of an initial denaturation step of $1 \mathrm{~min}$ at $95^{\circ} \mathrm{C}$, followed by 40 cycles of denaturation for $15 \mathrm{~s}$ at $95^{\circ} \mathrm{C}$, annealing for $20 \mathrm{~s}$ at $60^{\circ} \mathrm{C}$, and extension for $20 \mathrm{~s}$ at $72^{\circ} \mathrm{C}$. The real-time PCR thermal cycler qTOWER 2.0/2.2 (Analytik Jena, Jena, Germany) was used to obtain relative expression levels of each sample. All qRT-PCR expression assays were performed independently and analyzed three times under identical conditions. 


\section{RESULTS}

\section{Construction of the Gbvdr3 gene promoter and plant expression vector}

The 1.2-kb DNA fragment was obtained by two amplifications of genome walking PCR (Figure 1). Compared with the cDNA sequence of Gbvdr3, the 1.2-kb DNA fragment has 150 nucleotides in common with the target gene and partially overlaps with the same, suggesting that the fragment was the targeted gene promoter sequence (Gbvdr3) (Figure 1). The primers were designed according to the Gbvdr3 sequences and the recombinant vector of pBI-Gbvdr3-GUS (Figure 2) was constructed.

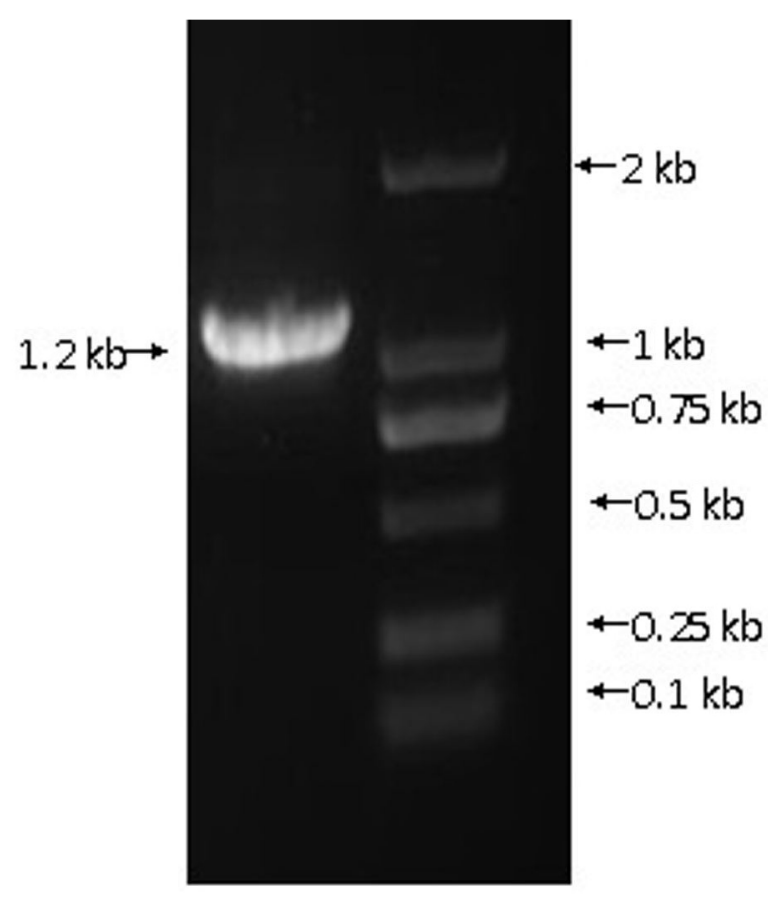

Figure 1. Promoter fragment of Gbvdr3 isolated by genome walking.

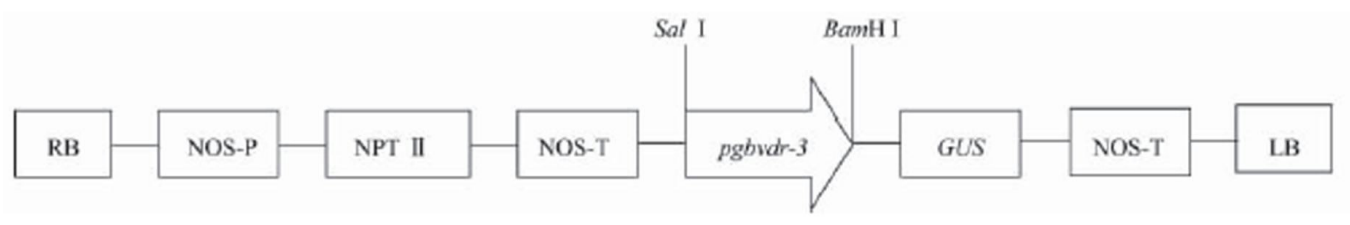

Figure 2. Diagram of pBI- Gbvdr3-GUS. 


\section{Gbvdr3 gene promoter sequence}

The Gbvdr3 promoter sequences were analyzed by the PLACE software (Figure 3 ). The promoter contained multiple light regulatory elements such as GATABOX (GATA) EBOXBNNAPA (CANNTG), GT1CONSENSUS (GRWAAW), IBOXCORE (GATAA), INRNTPSADB (YTCANTYY), and so on. Many researchers suggested that the disease resistance of plants is bound with light (Asai et al., 2000; Brodersen et al., 2002; Fryer et al., 2003). The Gbvdr3 promoter also contained a number of defense related components such as BIHD1OS (TGTCA), ELRECOREPCRP1 (TTGACC), GCCCORE (GCCGCC), HSELIKENTACIDICPR1 (CNNGAANNNTTCNNG), SEBFCONSSTPR10A(YTGTCWC), WBOXATNPR1 (TTGAC) and others, indicating that the promoter may be induced by pathogens. Furthermore, the promoter also contained multiple hormone response elements such as auxin and salicylic acid induction component ASF1MOTIFCAMV (TGACG), gibberellin induction component CAREOSREP1 (CAACTC), and ABA induction component DPBFCOREDCDC3 (ACACNNG). In addition, we also found many organ-specific components such as pollen-specific expression components POLLEN1LELAT52 (AGAAA), root hair-specific components RHERPATEXPA7 (KCACGW), and root expression element ROOTMOTIFTAPOX1 (ATATT).

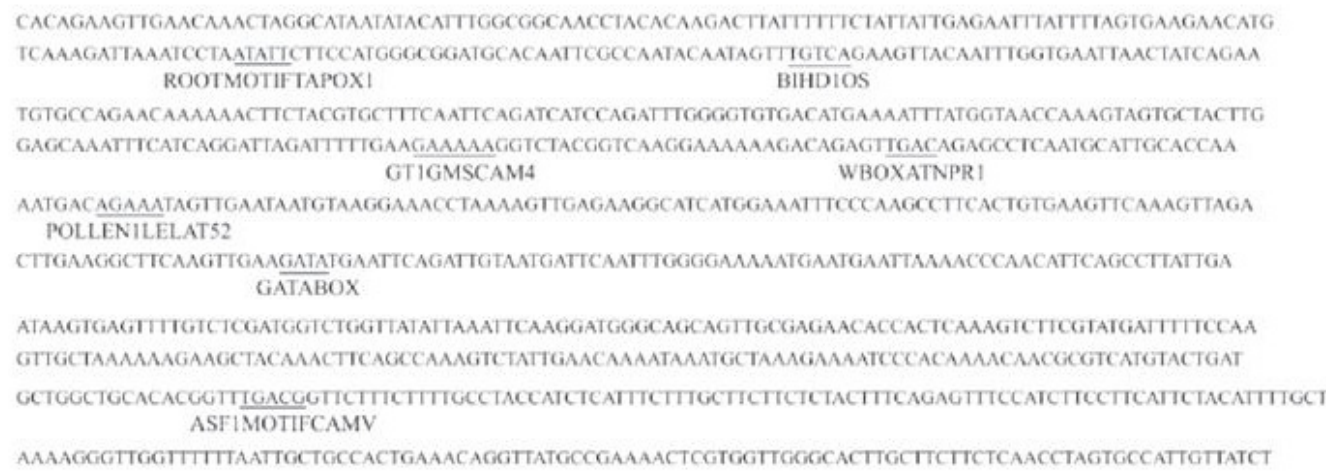

Figure 3. Sequence of the Gbvdr3 promoter.

\section{Transgenic Arabidopsis with Gbvdr3 promoter}

The 35S:Gbvdr3 construct was introduced into Arabidopsis ecotype Col-0 and eight independent, fertile primary Arabidopsis transformants were regenerated. The eight positive transgenic lines obtained by Agrobacterium-mediated transformation were confirmed by PCR (Figure 4).

\section{Expression pattern of the Gbvdr3 promoter in Arabidopsis}

After the GUS staining, the roots were dark in color, indicating that Gbvdr3 promoter was highly expressed in the roots (Figure 5A). Additionally, in other plant parts, Gbvdr3 promoter was significantly expressed in floral organs (style and stamens), there was 
no expression in the pod, and some level of expression was detected in seeds (Figure 5). Therefore, the Gbvdr3 promoter displayed a tissue-specific expression pattern. These plants exhibited constitutive GUS expression.

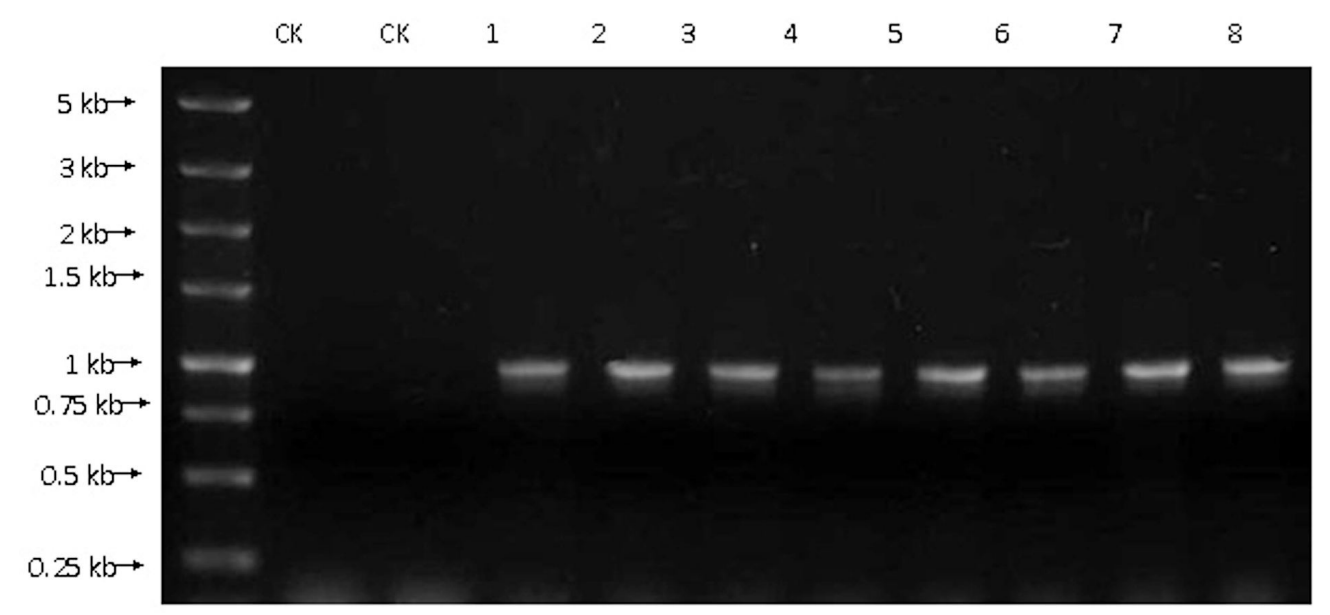

Figure 4. PCR identification of Gbvdr3 transgenic lines. CK, untransformed plants; 1-8, transformed plants.

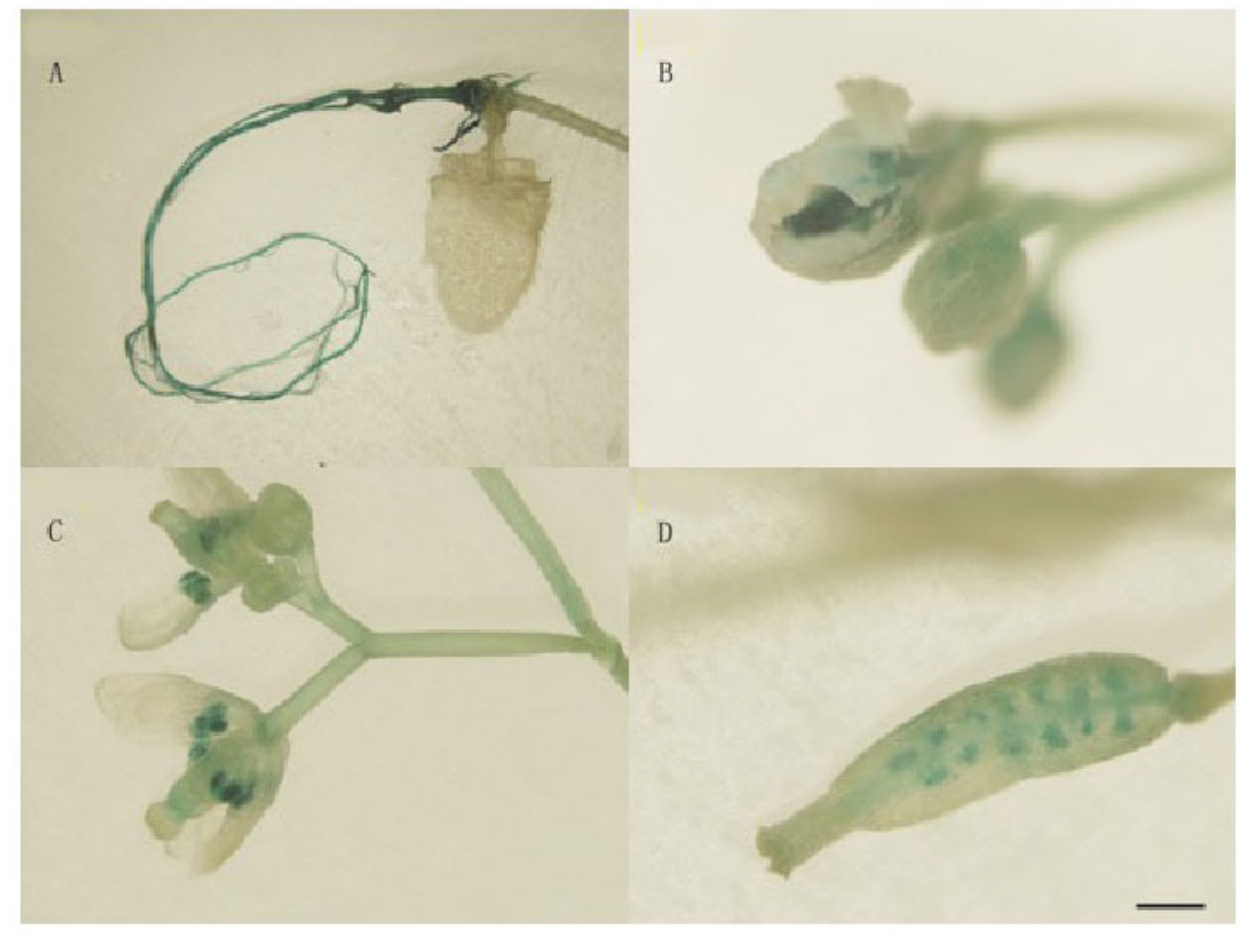

Figure 5. GUS expression in transgenic Arabidopsis. Bar $=100 \mathrm{~mm}$. A. root; B. C. flower; D. pod with seeds. 


\section{Expression of the Gbvdr3 gene in different organs and stages of $G$. barbadense development}

The results of the qRT-PCR showed that at the seedling stage expression of Gbvdr3 was higher in the leaves than in the root, and the lowest expression was detected in the stem at the seedling stage. At the flowering stage, expression of $G b v d r 3$ in the flower was higher than that in the stem, and it was the lowest in the flower bud (Figure 6).
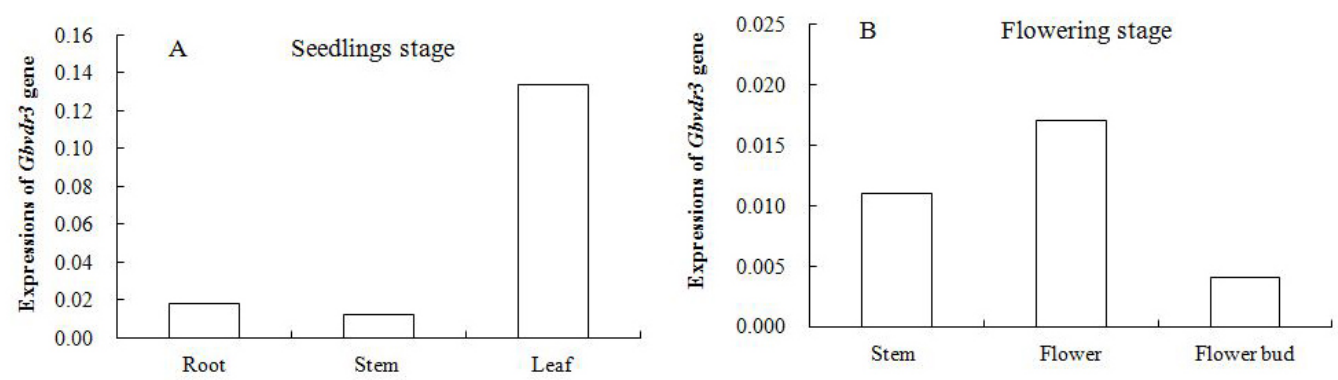

Figure 6. Expression of $G b v d r 3$ in different organs at different growth stages of the seedlings.

\section{DISCUSSION}

Efficient genetic modification of cotton for agronomic traits requires the use of regulatory sequences known as promoters that drive the expression of downstream transgenes in specific plant tissues. Currently, the widely used promoter for gene expression in dicotyledons including cotton is the cauliflower mosaic virus (CaMV) 35S promoter, which provides strong constitutive expression (Song et al., 2000). However, to develop transgenic cotton with Verticillium resistance, a larger arsenal of constitutive and tissue-specific promoters is required. The need for more developmentally or environmentally regulated promoters has become evident and considerable effort has been allocated into the discovery of specific tissue or biotic and hormonal or abiotic stress-responsive genes and promoters (Potenza and Aleman, 2004). Constitutive promoters of actin, ubiquitin, and tubulin gene have been widely used in various plant species for expressing transgenes or selectable markers, such as $R b c S$ gene promoter (Tanabe et al., 2015), potato tuber tissue-specific promoter of GBSS (Chen et al., 2011), and Arabidopsis thaliana pyk10 gene promoter (Lü et al. 2011). The Gbvdr3 promoter displayed a tissue-specific expression pattern. The transgenetic Arabidopsis exhibited constitutive GUS expression, light regulatory elements, defense related components, multiple hormone response elements, and organ-specific components (Chen et al., 2016).

Gbvdr3 and the reported $\mathrm{Vel}$ homologous genes in sea island cotton, including GbVe (Zhang et al., 2011), Gbvel (Zhang et al., 2012), and Gbvdr5 (Yang et al., 2014), suggest that the inheritance of cotton resistance to Verticillium dahliae may be controlled by multidominant resistant genes, which have a unique resistance specificity due to the recognition of different effectors. The promoter region (1.5 kb upstream) of the Gbvdr3 was cloned. The results of the histochemical staining confirmed that the Gus activity was specifically localized in the roots and flowers of the transgenic plants. These results indicate that Gbvdr3 can be 
induced by defense signaling molecules and infection by $V$. dahliae in vascular regions of the roots and stems. Gbvdr3 is regulated by some complex factors involved in cotton resistance and stress-induced processes (Zhao et al., 2013). Better understanding of the functional activity of the $G b v d r 3$ promoter important for further studyies of $G b v d r 3$ gene function.

In conclusion, the cloned promoter was fused to GUS reporter gene and transformed into Arabidopsis. Histochemical staining revealed that GUS expression was specifically detected in roots, flowers and seeds, indicating that $G b v d r 3$ is a tissue-specific promoter.

\section{ACKNOWLEDGMENTS}

Research supported by grants from the Natural Science Foundation of Jiangsu Province Youth Fund (\#SBK2015040083) and the Chinese Ministry of Agriculture through Transgenic Special Funds projects (\#2009ZX 08005-003B).

\section{REFERENCES}

Anne D and Steven EC (2004). LRR-containing receptors regulating plant development and defense. Development 31: 251-261.

Artico S, Nardeli SM, Brilhante O, Grossi-de-Sa MF, et al. (2010). Identification and evaluation of new reference genes in Gossypium hirsutum foraccurate normalization of real-time quantitative RT-PCR data. BMC Plant Biol. 10: 49. http://dx.doi.org/10.1186/1471-2229-10-49

Asai T, Stone JM, Heard E, Kovtun Y, et al. (2000). Fumonisin B1-induced cell death in Arabidopsis protoplasts requires jasmonate-, ethylene-,and salicylate-dependent signaling pathways. Plant Cell 12: 1823-1836. http://dx.doi. org/10.1105/tpc. 12.10 .1823

Belfanti E, Silfverberg Dilworth E, Tartarini S, Patocchi A, et al. (2004). The HcrVfa2 gene from a wild apple confers scab resistance to a transgenic cultivated variety. Proc. Natl. Acad. Sci. USA 101: 886-890. http://dx.doi.org/10.1073/ pnas.0304808101

Bradford MM (1976). A rapid sensitive method for the quantitation of microgram quantities of protein utilizing the principle of protein-dye binding. Anal. Biochem. 72: 248-254. http://dx.doi.org/10.1016/0003-2697(76)90527-3

Brodersen P, Petersen M, Pike HM, Olszak B, et al. (2002). Knockout of Arabidopsis ACCELERATED-CELL-DEATH11 encoding a sphingosine transfer protein causes activation of programmed cell death and defense. Genes Dev. 16: 490502. http://dx.doi.org/10.1101/gad.218202

Chen GL, Chen ZL and Qi XY (2011). GBSS of potato tuber tissue-specific promoter cloning and sequence analysis. Yangtze Vegetables 8: 25-27.

Chen T, Kan J, Yang Y, Ling X, et al. (2016). A Ve homologous gene from Gossypium barbadense, Gbvdr3, enhances the defense response against Verticillium dahliae. Plant Physiol. Biochem. 98: 101-111. http://dx.doi.org/10.1016/j. plaphy.2015.11.015

Fradin EF, Abd-El-Haliem A, Masini L, van-den-Berg GC, et al. (2011). Interfamily Transfer of Tomato Vel Mediates Verticillium Resistance in Arabidopsis. Plant Physiol. 156: 2255-2265. http://dx.doi.org/10.1104/pp.111.180067

Fryer MJ, Ball L, Oxborough K, Karpinski S, et al. (2003). Control of ascorbate peroxidase-2 expression by hydrogen peroxide and leaf water status during excess light stress reveals a functional organization of Arabidopsis leaves. Plant J. 33: 691-705. http://dx.doi.org/10.1046/j.1365-313X.2003.01656.x

Hua DP, Wang C, He J, Liao H, et al. (2012). A plasmamembrane receptor kinase, GHR1, mediates abscisic acid- and hydrogen peroxideregulated stomatal movement in Arabidopsis. Plant Cell 24: 2546-2561. http://dx.doi.org/10.1105/ tpc. 112.100107

Huang GT, Ma SL, Bai LP, Zhang L, et al. (2012). Signal transduction during cold, salt, and drought stresses in plants. Mol. Biol. Rep. 39: 969-987. http://dx.doi.org/10.1007/s11033-011-0823-1

Jefferson RA, Kavanagh TA and Bevan MW (1987). GUS fusions: beta-glucuronidase as a sensitive and versatile gene fusion marker in higher plants. EMBO J. 6: 3901-3907.

Jones DA, Thomas CM, Hammond-Kosack KE, Balint-Kurti PJ, et al. (1994). Isolation of the tomato Cf-9 gene for resistance to Cladosporium fulvum by transposon tagging. Science 266: 789-793. http://dx.doi.org/10.1126/ $\underline{\text { science. } 7973631}$ 
Kawchuk L, Hachey J and Lynch D (2001). Tomato Ve disease resistance genes encode cell surface-like receptors. Proc. Natl. Acad. Sci. USA 98: 6511-6515. http://dx.doi.org/10.1073/pnas.091114198

Lü SH, Sun KY and Fan YL (2011). The expression of Arabidopsis thaliana root-specific gene promoter in tobacco. Northwest Agric. Sci. 31: 1105-1109.

Malnoy M, Xu M, Borejsza-Wysocka E, Korban SS, et al. (2008). Two receptor-like genes, Vfal and Vfa2, confer resistance to the fungal pathogen Venturia inaequalis inciting apple scab disease. Mol. Plant Microbe Interact. 21: 448-458. http://dx.doi.org/10.1094/MPMI-21-4-0448

Nadeau JA and Sack FD (2002). Control of stomatal distribution on the Arabidopsis leaf surface. Science 296: 1697-1700. http://dx.doi.org/10.1126/science.1069596

Ouyang SQ, Liu YF, Liu P, Lei G, et al. (2010). Receptor-like kinase OsSIK1 improves drought and salt stress tolerance in rice (Oryza sativa) plants. Plant J. 62: 316-329. http://dx.doi.org/10.1111/j.1365-313X.2010.04146.x

Potenza CL and Aleman C (2004). Targeting transgene expression in research, agricultural, and environmental applications: promoters used in plant transformation. In Vitro Cell. Dev. Biol. Plant 40: 1-22. http://dx.doi.org/10.1079/IVP2003477

Ramonell K, Berrocal-Lobo M, Koh S, Wan J, et al. (2005). Loss-of-function mutations in chitin responsive genes show increased susceptibility to the powdery mildew pathogen Erysiphe cichoracearum. Plant Physiol. 138: 1027-1036. http://dx.doi.org/10.1104/pp.105.060947

Ron M and Avni A (2004). The receptor for the fungal elicitor ethylene-inducing xylanase is a member of a resistance-like gene family in tomato. Plant Cell 16: 1604-1615. http://dx.doi.org/10.1105/tpc.022475

Song P, Heinen JL and Burns TH (2000). Expression of two tissue-specific promoters in transgenic cotton plants. J. Cotton Sci. 4: 217-223.

Taguchi-Shiobara F, Yuan Z, Hake S and Jackson D (2001). The fascinated ear2 gene encodes a leucine-rich repeat receptor-like protein that regulates shoot meristem proliferation in maize. Genes Dev. 15: 2755-2766. http://dx.doi. org/10.1101/gad.208501

Tanabe N, Tamoi M and Shigeoka S (2015). The sweet potato RbcS gene (IbRbcS1) promoter confers high-level and green tissue-specific expression of the GUS reporter gene in transgenic Arabidopsis. Gene 567: 244-250. http:// dx.doi.org/10.1016/i.gene.2015.05.006

Tanaka H, Osakabe Y, Katsura S, Mizuno S, et al. (2012). Abiotic stress-inducible receptor-like kinases negatively control ABA signaling in Arabidopsis. Plant J. 70: 599-613. http://dx.doi.org/10.1111/j.1365-313X.2012.04901.x

Yang Y, Ling X, Chen T, Cai L, et al. (2014). A cotton Gbvdr5 gene encoding a leucine-rich-repeat receptor-like protein confers resistance to Verticillium dahliae in transgenic Arabidopsis and upland cotton. Plant Mol. Biol. Rep. 33: 1-15.

Zhang B, Yang Y, Chen T, Yu W, et al. (2012). Island cotton gbve1 gene encoding a receptor-like protein confers resistance to both defoliating and non-defoliating isolates of Verticillium dahliae. PLoS One 7: e51091. http://dx.doi. org/10.1371/journal.pone.0051091

Zhang Y, Wang X, Yang S, Chi J, et al. (2011). Cloning and characterization of a Verticillium wilt resistance gene from Gossypium barbadense and functional analysis in Arabidopsis thaliana. Plant Cell Rep. 30: 2085-2096. http://dx.doi. org/10.1007/s00299-011-1115-x

Zhao J, Gao YL, Zhang ZY, Chen TZ, et al. (2013). A receptor-like kinase gene (GbRLK) from Gossypium barbadense enhances salinity and drought-stress tolerance in Arabidopsis. BMC Plant Biol. 13: 110. http://dx.doi. org/10.1186/1471-2229-13-110 